\title{
Health Risk Tolerance as A Key Determinant of (Un)willingness to Behavior Change: Conceptualization and Scale Development
}

\author{
Hyoyeun Jun \\ University of Georgia \\ Athens, Georgia USA \\ Yan Jin \\ University of Georgia \\ Athens, Georgia USA
}

\begin{abstract}
After the study of testing determinants of risk tolerance affecting information sharing, this study was conducted as a second step to actually develop the scale for risk tolerance. Firstly, this study followed qualitative steps, such as in-depth interview and focus group, to capture how public describes the situation when they are tolerating the risk, when they knew what the recommended behavior is to relieve the risk. Secondly, this study collected 1000 U.S. public sample for the survey questionnaire that are the items generated from the qualitative steps.
\end{abstract}

Keywords: Risk Communication, Risk Tolerance, Scale Development

SUGGESTED CITATION: Jun, H., \& Jin, Y. (2019). Health risk tolerance as a key determinant of (un)willingness to behavior change: conceptualization and scale development. Proceedings of the International Crisis and Risk Communication Conference, Volume 2 (pp. 18-19). Orlando Fl: Nicholson School of Communication and Media. https://doi.org/10.30658/icrcc.2019.5

\section{INTRODUCTION}

As Heath and O'Hair (2009) defined, crisis is when risk is manifested. The urgency and uncertainty of crisis can induce more complexity to organizations [2] Public health crisis and risk communications are tasked to communicate about risks that can harm public health and persuade the public to adopt healthier and less risky behavior [1] Although risk perception itself has been extensively studied, it remains unclear how individuals choose not to modify unhealthy behaviors despite their awareness of the benefits of changing such behaviors. To further unearth the psychological process of refusal to change, our study introduces and explicates the concept of risk tolerance as a key determinant of individuals' (un)willingness to modify unhealthy behaviors.

\section{LITERATURE REVIEW AND METHODS}

Risk tolerance, a concept originally developed in management and financial planning, is defined in our study as how much individuals tolerate not to follow the recommended healthy behavior. To refine the conceptualization and develop a scale measuring health risk tolerance, a multi-phase, multi-method research design is employed.

Phase I: A focus group of 30 college students and 28 in-depth interviews with non-student adults were conducted to generate potential items to be included in risk tolerance scale, based on actual descriptors people use to indicate how they tolerate health risks, when they are aware of the benefits of changing unhealthy behavior but still choose not to modify their behavior. A total 
of 55 items 1 were generated for initial scale development. Examples of popular items, emerged in both the focus group and across in-depth interviews, include: "I understand that there is higher risk if I keep doing this and not following the recommended behavior but I had to do this anyways", "I keep putting it off to follow that recommended behavior", "I do not mind taking the risk of not following the recommended behavior", "I just ignore the recommended healthier choice, because it does not affect me", "I know that I will eventually follow the advice, but just for now, where deep down I know that I probably will not follow that advice", etc. Our results also indicate that the level of health risk tolerance vary depending on the type of comparison, the existence of higher goal, temporal distance of potential risks, economic barriers to follow the recommended behavior.

Phase II: Online surveys are conducted among college students and non-student adult samples, to test the structure, validity, and reliability of the health risk tolerance scale, using exploratory factor analyses (EFAs) and confirmatory factor analyses (CFAs). The data collection and analyses will be completed by the end of May 2019.

\section{DISCUSSION}

The concept and the scale of health risk tolerance provide a comprehensive picture of how individuals tolerate health risks and a measurement tool to gauge it systematically. Our findings imply opportunities for crisis and risk communication professionals to develop persuasion strategies targeting at decreasing health risk tolerance, thus increasing individuals' willingness to follow recommended preventive or protective actions and modify their unhealthy or unsafe behaviors accordingly.

\section{Author Biography}

Hyoyeun Jun (hj15075@uga.edu) is a Ph.D. candidate at the Grady College of Journalism and Mass Communication in the University of Georgia.

Yan Jin, Ph.D. (yanjin@uga.edu) is an assistant department head; associate director of Center for Health \& Risk Communication; Athletic Association Professor in Grady College; Professor in Public Relations at the Grady College of Journalism and Mass Communication in the University of Georgia.

\section{REFERENCES}

[1] Heath, R. L., \& O’Hair, H. D. (2009). The significance of crisis and risk communication. In R. L. Heath \& H. D. O’Hair (Eds.), Handbook of crisis and risk communication (pp.1-30). New York: Routledge.

[2] Freimuth, V., Linnan, H. W., \& Potter, P. (2000). Communicating the Threat of Emerging Infections to the Public. Emerging Infectious Diseases, 6, 337-347. https://doi.org/10.3201/eid0604.000403

[3] Seeger, M. W., Sellnow, T. L., \& Ulmer, R. R. (1998). Communication, organization, and crisis. Annals of the International Communication Association, 21, 231-276. https://doi.org/10.1080/23808985.1998.11678952 